\title{
Large Aperture Mirror Surface Test
}

\author{
Hui Xing, Ye Zhao, Junru Song \\ Key Laboratory for Advanced Optical Remote Sensing Technology of Beijing, Beijing Institute of Space Mechanics \& Electricity, \\ Beijing, China \\ Email:11158804@qq.com
}

How to cite this paper: Xing, H., Zhao, $\mathrm{Y}$. and Song, J.R. (2021) Large Aperture Mirror Surface Test. Optics and Photonics Journal, 11, 387-393. https://doi.org/10.4236/opj.2021.118027

Received: June 29, 2021

Accepted: August 20, 2021

Published: August 23, 2021

\begin{abstract}
The large aperture mirror surface test is the basis of optical processing and alignment, and is also the key to the development of remote sensing device. The simulation results show that the RMS values of $1.07 \mathrm{~m}$ primary mirror with multi-point support and sling support are $1.86 \mathrm{~nm}$ and $3.28 \mathrm{~nm}$ respectively. Using 36 point unloading device, sponge 36 point free support and sling support to test the mirror surface, the results are basically consistent, RMS is better than $0.02 \lambda(\lambda=632.8 \mathrm{~nm})$.
\end{abstract}

\section{Keywords}

Large Aperture Mirror, Surface Test, Support

\section{Introduction}

With the rapid development of the field of ground observation, deep space exploration and scientific experiment, the resolution requirements of space remote sensing cameras are also higher and higher. Because the angular resolution of optical system is inversely proportional to the aperture of the through-beam, increasing the aperture of space remote sensor is the most direct and effective means to improve the resolution. As the largest mirror in optical system, how to keep the surface stability is the primary engineering problem in the development of remote sensing camera. The primary mirror surface will deviate from the real surface due to the influence of gravity deformation and support deformation during the ground detection. How to remove the influence of gravity and support is the key to surface test [1] [2].

\section{Principle Analysis}

There are two common methods in the measurement of large aperture mirror surface, one is multi-point unloading support and the other is sling support. The 
principle analysis of two test methods is given below.

\subsection{Multi Point Unloading Support}

Because the mirror cannot be an absolute rigid body, according to the elastic deformation principle, with the increase of the mirror aperture, the structural stiffness of the mirror itself will gradually decrease, and the number of supporting points must be increased to ensure the accuracy of the mirror surface. The elastic deformation analysis of the surface under the equal spacing support is shown in Figure 1. G is the acceleration of gravity, and D is the spacing of support points.

It can be seen that the surface of the mirror is proportional to the Fourth Square of the distance D of the support point, and is inversely proportional to Young's modulus and moment of inertia. Therefore, in order to reduce the distortion of mirror, only one way to reduce the support distance is to increase the number of support points when the mirror structure and material are determined.

When the optical axis is vertically supported and unloaded, the force of the mirror shall be balanced, so as to prevent the movement in the space of the mirror, namely, it meets the following requirements:

$$
\sum_{i=1}^{36} F_{i}-M_{m} g=0
$$

The mirror must meet the moment balance, so as to prevent the rotation in the mirror space, namely, it meets the following requirements:

$$
\sum_{i=1}^{36} M_{i}=0
$$

When the optical axis is vertical (pointing to the roof), 36 back supporting points are designed on the back of the mirror, and the supporting diameter is taken $\Phi 25 \mathrm{~mm}$ (Figure 2).

When the optical axis is vertical, the mirror is affected by its own weight, and the surface is shown in Figure 3, RMS is $1.86 \mathrm{~nm}$.

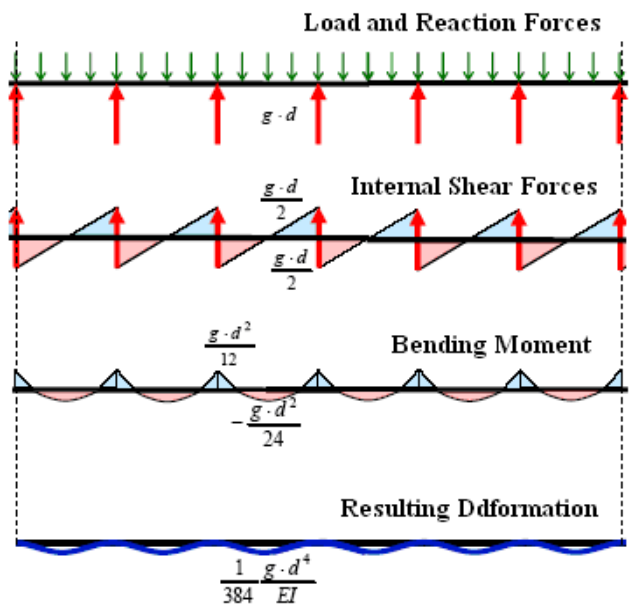

Figure 1. Deformation analysis. 


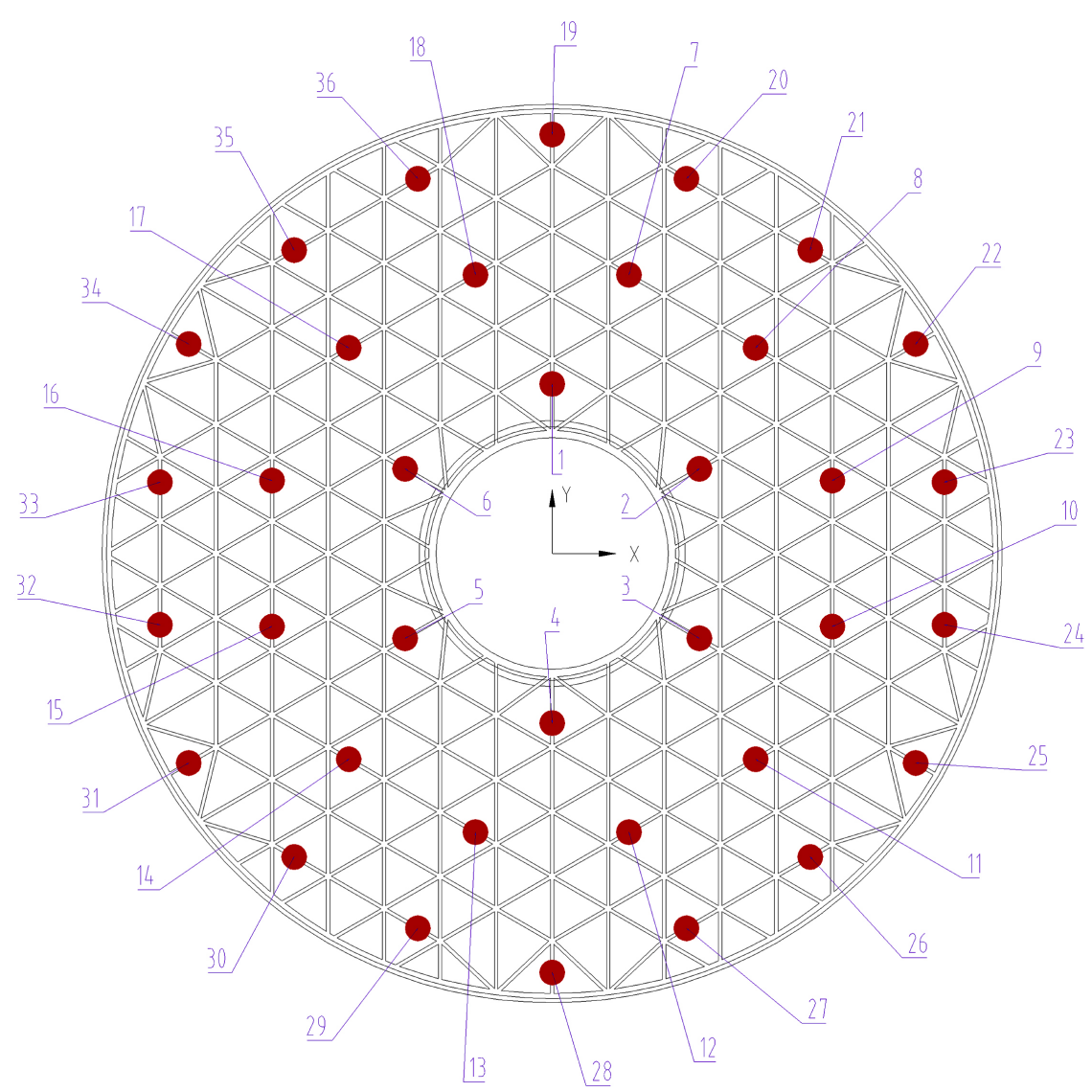

Figure 2. Distribution of support at 36 points on the back of mirror.

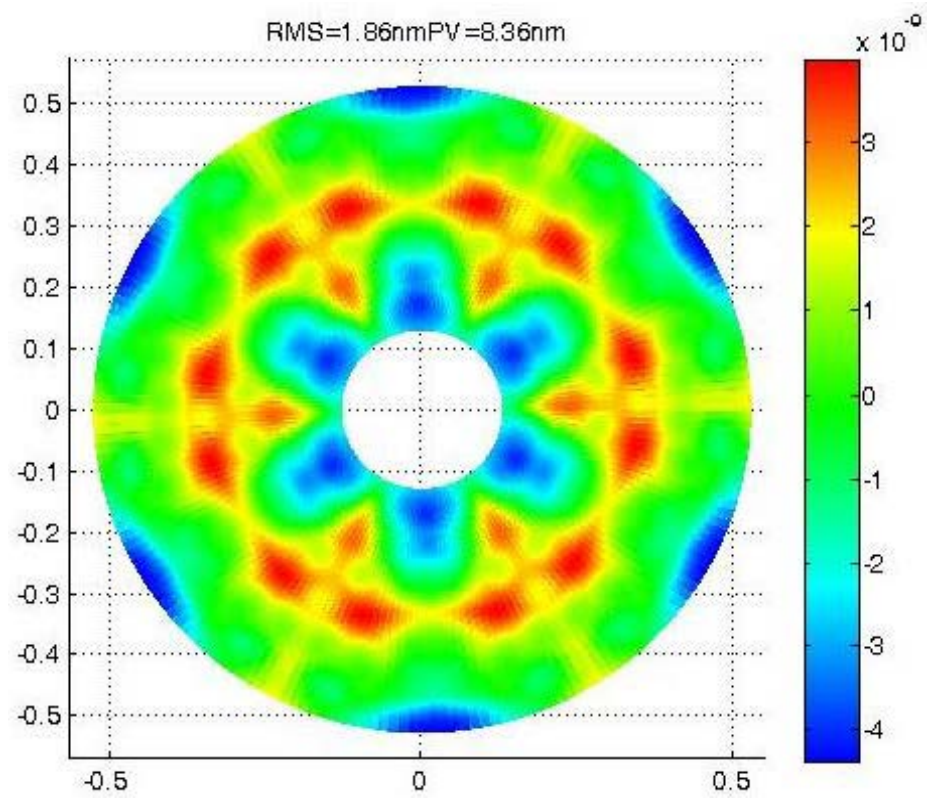

Figure 3. Mirror surface when the optical axis is vertical.

\subsection{Sling Support (Figure 4)}

When the optical axis of the primary mirror is horizontal, there are two kinds of 

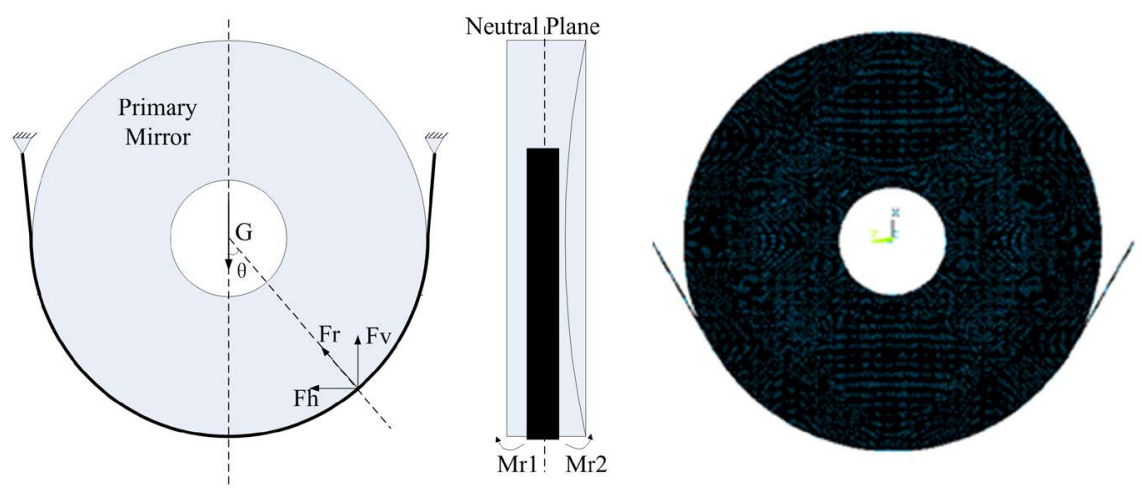

Figure 4. Schematic diagram of the stress state of sling support and mesh generation.

forces supported by sling. One is the support force Fr of the sling to the mirror along the radial direction. The set $\mathrm{Fv}$ of its components is balanced with gravity and distributed along the consine around the primary mirror. The left and right symmetrical components Fh balance each other. The second is that the bending moment $\mathrm{Mr} 1$ and $\mathrm{Mr} 2$ of the mirror under the support of the edge of the sling reach the balance. The bending moment is more sensitive to the surface of the mirror.

The mesh model of the primary mirror is established by the same method as that of linear analysis. The sling is divided by shell element, and the contact pair is established in the contact area between the outer ring of primary mirror and sling. The parameters of sling are: elastic modulus $140 \mathrm{Gpa}$, Poisson ratio 0.3, density $8100 \mathrm{~kg} / \mathrm{m}^{3}$, width $80 \mathrm{~mm}$, thickness $1 \mathrm{~mm}$. The friction coefficient between the sling and the primary mirror is 0.5 . Through simulation analysis, the gravity deformation of the primary mirror surface $\mathrm{rms}=3.28 \mathrm{~nm}$, and the cloud diagram of gravity deformation of primary mirror is shown in Figure 5.

\subsection{Aberrations Expression of Detection System}

The optical component of the detection system is mainly introduced into primary aberrations, and no new aberrations will be generated. The Zernike scalar expression of the primary aberrations of the system is as follows [3] [4]:

$$
W_{j}(\rho, \phi)=\sum_{j=5}^{9} C_{i}^{j} Z_{i}(\rho, \phi)
$$

$W_{j}(\rho, \phi)$ is the aberration of the field of view, $Z_{i}(\rho, \phi)$ is Zernike polynomial and $C_{i}^{j}$ is the polynomial field fitting coefficient. Table 1 gives the expressions of the astigmatism, coma and spherical aberration of polynominal expressions, $\theta$ is the polar angle, $\rho$ is the radius.

\section{Measurement}

\subsection{Unloading Device 36 Point Support}

The self collimation optical path as shown in Figure 6 is established. After passing through the compensator and interferometer, the parallel light from the 


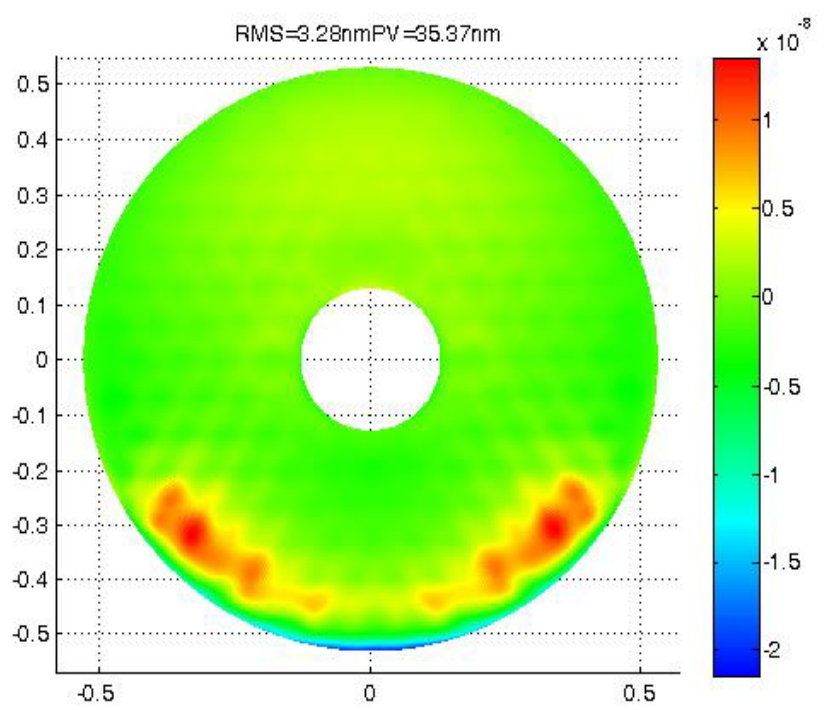

Figure 5. Nonlinear analysis surface of sling.

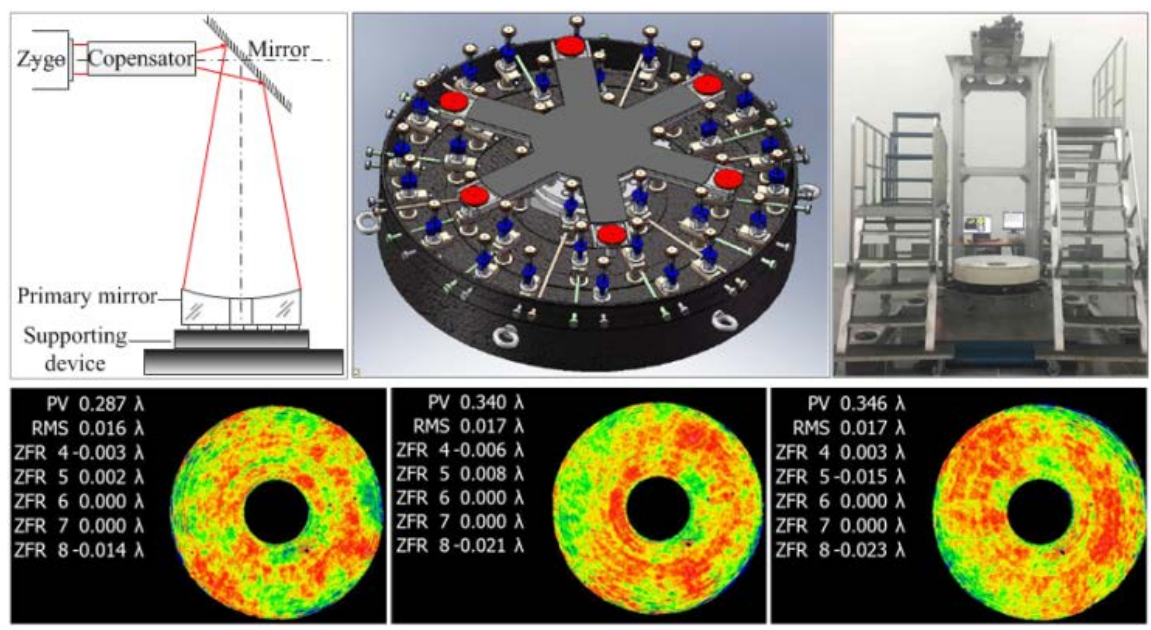

Figure 6. 36 point unloading support and test results.

Table 1. Zernike polynomial expressions.

\begin{tabular}{ccc}
\hline Polynomial expression & Meaning & Brief exp. \\
\hline$\rho^{2} \cos 2 \theta$ & Astigmatism $0^{\circ}$ or $90^{\circ}$ & $Z_{5}$ \\
$\rho^{2} \sin 2 \theta$ & Astigmatism $\pm 45^{\circ}$ & $Z_{6}$ \\
$\left(3 \rho^{2}-2\right) \rho \cos \theta$ & X Coma and Tilt & $Z_{7}$ \\
$\left(3 \rho^{2}-2\right) \rho \sin \theta$ & Y Coma and Tilt & $Z_{8}$ \\
$6 \rho^{4}-6 \rho^{2}+1$ & Spherical and Focus & $Z_{9}$ \\
\hline
\end{tabular}

interferometer reaches the primary mirror and collimated. According to 1.1, 36 supporting tooling are designed, and the moment of each support point is calculated. After measuring a position, taking the current position as the zero position, the primary mirror rotates $120^{\circ}$ and $240^{\circ}$ around the optical axis respec- 
tively. The results of the three positions are shown in Figure 6. It can be seen that when the primary mirror is at zero, $120^{\circ}$ and $240^{\circ}$, the test results are consistent.

\subsection{Sponge 36 Point Support}

As is shown in Figure 7, paste sponge with diameter of $50 \mathrm{~mm}$ and thickness of $60 \mathrm{~mm}$ on aluminum plate with diameter of $1300 \mathrm{~mm}$, thickness of $20 \mathrm{~mm}$ and flatness of $0.02 \mathrm{~mm}$. Put the primary mirror with $1.07 \mathrm{~m}$ diameter on the sponge. Adjust the compensator and the plane mirror to establish the autocollimation optical path. It can be seen that the surface test results of the primary mirror are consistent with the results of the 36 point unloading device under the free support of sponge.

\subsection{Sling Support}

As shown in Figure 8, the primary mirror is tested by sling, and Fv shall pass the center of gravity of the mirror. After measuring the current position surface, the primary mirror rotates $120^{\circ}$ and $240^{\circ}$ around the optical axis respectively. It can be seen that the test results are consistent with the optical axis vertical unloading support results.
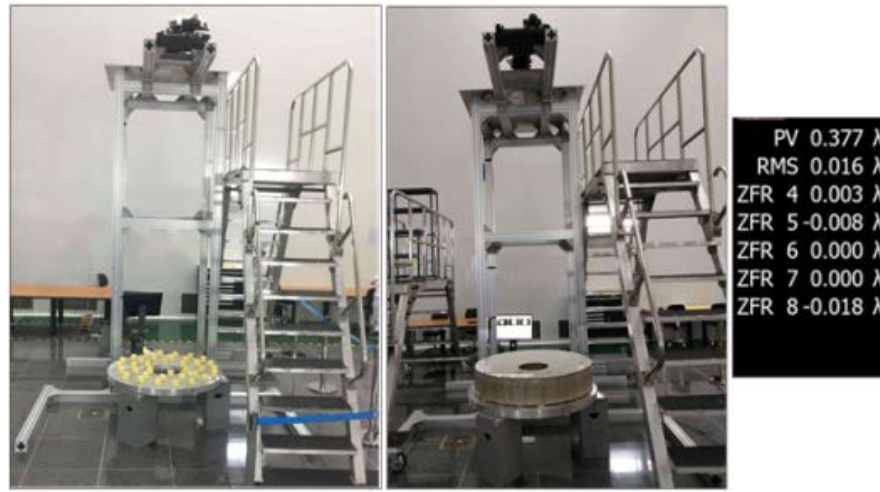

RMS $0.016 \lambda$

R $40.003 \lambda$

$\begin{array}{lll}\text { ZFR } & 5 & -0.008 \lambda \\ \text { ZFR } & 6 & 0.000\end{array}$

ZFR $70.000 \lambda$

ZFR $8-0.018 \lambda$

Figure 7. Sponge free support and test results.

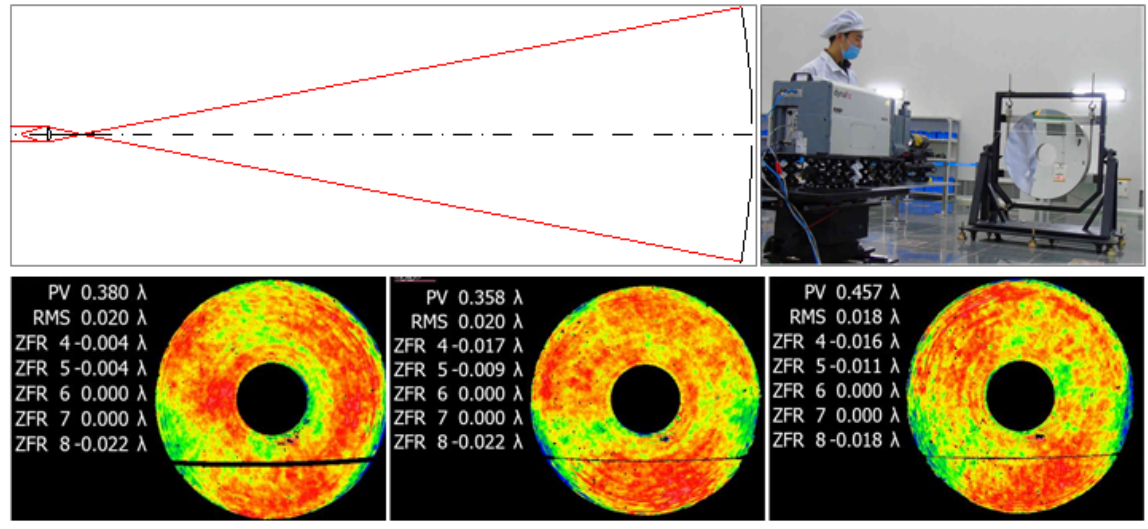

Figure 8. Sling support and test results. 


\section{Conclusion}

Based on the analysis of the horizontal and vertical stress states of the optical axis of the large caliber primary mirror, the simulation results of the surface of the primary mirror under different supporting conditions are given. The primary mirror surface is tested by using multi-point support and sling support, and the test results are basically the same. The results of multi-point unloading support can be used as the basis for optical processing of mirrors, and the results of sling support can be used as the basis for structural design and optical alignment.

\section{Conflicts of Interest}

The authors declare no conflicts of interest regarding the publication of this paper.

\section{References}

[1] Han, G., Cao, L. and Gao, Y. (2012) Supporting and Assembling for Primary Mirrors of $1 \mathrm{~m}$ Aperture Telescopes. Optics and Precision Engineering, 20, 1922-1927. https://doi.org/10.3788/OPE.20122009.1922

[2] Meng, X., Wang, Y. and Li, W. (2019) Fabrication of Zero-Gravity Surface for Large-Aperture Aspherical Mirror by Using Rotationally Method. Optics and Precision Engineering, 27, 2517-2522. https://doi.org/10.3788/OPE.20192712.2517

[3] Guo, P. and Yu, J. (2006) Improving Precision of Null Lens Method with Correction Technique. Optics and Precision Engineering, 14, 204.

[4] Wang, C., Huang, Y. and Wang, C. (2020) High Precision Reconstruction and Adjustment Technique for Surface Figure Error of the Off-Axis Reflective System. Spacecraft Recovery \& Remote Sensing, 41, 58-61. 